\title{
Modeling, Simulation, and Kinetic Studies of Solvent-Free Biosynthesis of Benzyl Acetate
}

\author{
Vijay Kumar Garlapati, Annapurna Kumari, Paramita Mahapatra, and Rintu Banerjee
}

Microbial Biotechnology and Downstream Processing Laboratory, Agricultural and Food Engineering Department, Indian Institute of Technology, Kharagpur, West Bengal 721302, India

Correspondence should be addressed to Rintu Banerjee; rb@iitkgp.ac.in

Received 15 May 2013; Accepted 28 August 2013

Academic Editor: Jose M. Cruz

Copyright (C) 2013 Vijay Kumar Garlapati et al. This is an open access article distributed under the Creative Commons Attribution License, which permits unrestricted use, distribution, and reproduction in any medium, provided the original work is properly cited.

\begin{abstract}
Solvent-free biosynthesis of benzyl acetate through immobilized lipase-mediated transesterification has been modeled and optimized through statistical integrated artificial intelligence approach. A nonlinear response surface model has been successfully developed based on central composite design with transesterification variables, namely, molarity of alcohol, reaction time, temperature, and immobilized lipase amount as input variables and molar conversion (\%) as an output variable. Statistical integrated genetic algorithm optimization approach results in an optimized molar conversion of $96.32 \%$ with the predicted transesterification variables of $0.47 \mathrm{M}$ alcohol molarity in a reaction time of $13.1 \mathrm{~h}$, at $37.5^{\circ} \mathrm{C}$ using $13.31 \mathrm{U}$ of immobilized lipase. Immobilized lipase withstands more than $98 \%$ relative activity up to 6 recycles and maintains $50 \%$ relative activity until 12 recycles. The kinetic constants of benzyl acetate, namely, $K_{m}$ and $V_{\max }$ were found to be $310 \mathrm{mM}$ and $0.10 \mathrm{mmol} \mathrm{h}^{-1} \mathrm{~g}^{-1}$, respectively.
\end{abstract}

\section{Introduction}

Flavour esters of short chain carboxylic acids and alcohols are among the most important and versatile components of natural flavors and fragrances and have a wide range of applications in food, beverage, cosmetic, and pharmaceutical sectors [1]. Knowing the health prospects of natural flavours, most of the customers are inclining towards the daily commodities which utilize biologically derived flavours (considered as "Natural Flavours") instead of chemical ones [2]. Among different biotechnological processes, immobilized lipase-mediated transesterification under solvent-free conditions is one of the economically viable clean technology for flavor ester production with continuous mode of operation $[3,4]$. The specificity of lipase, usage of mild reaction tempeartures, and solvent-free conditions making this process as immense commercial of interest and replacing the existed chemical catalyzed flavour ester synthesis [5]. Benzyl acetate is a short chain flavor ester found in plants such as jasmine, hyacinth, gardenias, and azaleas with the commercial value in flavour, food, and chemical industries. The global demand for benzyl acetate ranges between 5000 and 10,000 tons per annum. Covalent immobilization of lipases to insoluble polymeric supports aids in the industrial process economics by facilitating easy enzyme recovery from the reaction mixture [6]. The regio- and stereospecificities of immobilized lipase-mediated transesterification reactions enable the production of certain flavour compounds which are difficult to synthesize by chemical means in a sustainable manner and the products are considered as "natural." Several researchers reported the utilization of these catalysts (immobilized lipases) for flavour ester synthesis. Some attempts have been also made for the synthesis of benzyl acetate in solventassociated approach through chemical and biotechnological approaches. Amarnath et al. 2004 [7] reported the synthesis of benzyl acetate using polyaniline salts ( $\mathrm{Sb} / \mathrm{Pd}$ ratio of 0.7 ). Majumder et al. 2006 [8] utilized hexane as solvent, Lipozyme RM IM for the synthesis of benzyl acetate and investigated the effect of enzyme amount and substrate molar ratio on benzyl acetate yield. Synthesis of benzyl acetate under solvent-free conditions facilitate, the simplification of the downstream processing and reduced environmental hazards, and reduction of separation costs due to the nonrequirement of solvent recovery $[9,10]$. In lipase-mediated synthesis of flavour esters, 
the transesterification variables play an important role on the final ester yield. Moreover, the reaction side product water hampers the maximum ester yield by promoting the reverse reaction, hydrolysis. To attain the optimal yields of these flavour esters, having the knowledge of individual and interaction effects of transesterification variables on ester yield will be helpful to model and optimize the process.

In recent days, several researchers acknowledged the better solvable approaches of statistical integrated evolutionary optimization techniques in modeling and optimization of different bioprocesses. This integrated approach works on the statistical-based response surface methodology (RSM) and artificial intelligence-based Genetic algorithm (GA) approach [11]. RSM is a powerful mathematical modeling approach which does not need the explicit expressions of the physical meaning of the system or process under investigation and develops nonparametric regression model. This model approximates the functional relationships between transesterification variables (Input) and the molar conversion (output and response) of the process using experimental data [12] and helpful to estimate the optimal settings of input variables to maximize the response. Artificial intelligence based GA, developed by Holland, uses the Darwinian evolution concept of "survival of the fittest" to overcome the local optima obstacle by attaining the global optima quickly [13]. There have been a few attempts of using only statistical techniques in lipase-mediated synthesis of flavour esters to model and optimize the process but no attempts have been found using statistical integrated evolutionary approaches. A statistical approach was utilized to optimize the lipase. Palatase catalyzed biosynthesis of flavour-active octanoic acid esters from coconut cream and fusel oil as the biocatalyst with the influential significance of temperature, time, and enzyme amount on the ester yield $[14,15]$. Esterification reactions of butyl acetate and isoamyl acetate synthesis catalyzed by Candida antarctica lipase B (Novozym 435) were also optimized using response surface methodology [16, 17].

Hence, in the present study, the statistical integrated artificial intelligence was utilized for the first time in modeling and optimization of the immobilized lipase catalyzed synthesis of food flavour ester under solvent-free conditions. The significance of individual and combined effects of various transesterification variables, namely, molarity of alcohol, reaction time, temperature and immobilized enzyme amount on molar conversion was studied through response surface methodology and optimal conditions of the process was determined through GA approach. The reusability and kinetics of immobilized lipase in solvent-free system were also investigated.

\section{Materials and Methods}

2.1. Microorganism and Chemicals. A well-known lipolytic fungal strain Rhizopus oryzae 3562 was isolated from the local soil of IIT Kharagpur and maintained on Potato Dextrose Agar (PDA) medium. $p$-nitrophenyl panmitate ( $p$-NPP) and benzyl acetate standards were purchased from Sigma (USA). All chemicals used were of AR grade and were procured from Merck, Qualigens, and Himedia, India
2.2. Production and Immobilization of Lipase. Lipase was produced using wheat bran as a substrate through solid-state fermentation [18]. Lipase immobilization was carried out using covalent attachment technique using activated silica as immobilization matrix and was explained in our previous work [19].

2.3. Lipase Assay and Protein Determination. Lipase assay was done spectrophotometrically using pNPP as the substrate [20]. One unit (U) of enzyme is defined as the amount of enzyme that liberates one micromole of $p$-nitrophenol per minute under the assay conditions. Total protein was estimated using bovine serum albumin (BSA) as standard. [21].

2.4. Transesterification Reaction. Transesterification reaction was carried out in screw-capped vials containing benzyl alcohol in vinyl acetate $(0.3-0.7 \mathrm{M})$, where vinyl acetate acts as an acyl donor. Reaction was initiated by the addition of immobilized $R$. oryzae 3562 lipase (8-16 U). Samples were placed in an orbital shaker at $200 \mathrm{rpm}$ and temperatures (30$50^{\circ} \mathrm{C}$ ) for a time interval of $8-16 \mathrm{~h}$, along with the respective controls (based on the central composite design and one variable at time experiments).

2.5. GC Analysis. Aliquots of reaction mixture were withdrawn periodically from reaction mixture and centrifuged at $1747 \mathrm{~g}$ for $10 \mathrm{~min}$ to remove the immobilized enzyme then diluted with $n$-hexane (10 times). Synthesis of benzyl acetate was analyzed by injecting the diluted aliquots of the reaction mixture in a gas chromatograph (Agilent 6820). The column temperature was kept at $100^{\circ} \mathrm{C}$ for $1 \mathrm{~min}$, thereafter raised to $180^{\circ} \mathrm{C}$ at the rate of $15^{\circ} \mathrm{C} / \mathrm{min}$ and maintained at this temperature for $2 \mathrm{~min}$. The temperatures of both the injector and detector were set at $250^{\circ} \mathrm{C}$. Nitrogen was used as a carrier gas. The retention time of benzyl alcohol and benzyl acetate was $3.2 \mathrm{~min}$, and $4.4 \mathrm{~min}$ respectively.

2.6. Modelling Studies through RSM. Response surface methodology (RSM) is a statistical model approach for empirical modeling which evaluates the effect of individual and interaction effects of the process parameters on the corresponding response value. These effects can be approximated by the quadratic model equation through a sequence of designed experiments [22]. Transesterification reaction variables such as molarity of alcohol $(\mathrm{M})$, reaction time $(\mathrm{h})$, temperature $\left({ }^{\circ} \mathrm{C}\right)$, and immobilized enzyme amount (U) play an important role in solvent-free synthesis of Benzyl acetate. The individual and interaction effects of these parameters on the flavor ester yield in terms of molar conversion (\%) were studied by means of a central composite design (with four variables at three levels) of RSM using MINITAB 14 software. Range of these transesterification was chosen through the one variable at a time experiments and tabulated in Table 1 . The set of experiments executed was shown in Table 2 . Results were analyzed through the MINITAB 14 programs through the analysis of variance (ANOVA) and significance tests, and obtained RSM model was checked for adequacy through coefficient of determination $\left(R^{2} \&\right.$ adjusted $\left.R^{2}\right)$ and deleted residuals. 
TABLE 1: Transesterification variables and their levels.

\begin{tabular}{lcccc}
\hline \multirow{2}{*}{ Variable } & \multicolumn{4}{c}{ Levels } \\
& Notation & Low $(-1)$ & Center $(0)$ & High $(+1)$ \\
\hline Alcohol molarity $(\mathrm{M})$ & $X_{1}$ & 0.3 & 0.5 & 0.7 \\
Reaction time $(\mathrm{h})$ & $X_{2}$ & 8 & 12 & 16 \\
Temperature $\left({ }^{\circ} \mathrm{C}\right)$ & $X_{3}$ & 30 & 40 & 50 \\
Enzyme activity $(\mathrm{U})$ & $X_{4}$ & 8 & 12 & 16 \\
\hline
\end{tabular}

\subsection{Artificial Intelligence-Based Optimization Approach.} Simulation of the transesterification process through GA approach provides an accurate predicted result against the usual gradient-based optimization approaches. The parallel search pattern of GA makes the optimization task of choice for various industrial problems. In the present approach of binary coded GA (works on the mechanics of coping of the strings), three operations, namely, reproduction, crossover $\left(p_{c}\right)$, and mutation $\left(p_{m}\right)$ play an important role in creating a better solution. At first, the optimization approach starts with a randomly generated initial population $(N)$ of strings (in the form of binary coded individuals). The resultant fitness of each individual string after each generation is evaluated with respect to the given molar conversion (objective function) using the reproduction operator. At the end of each cycle, a mating pool of good strings was selected from the fitness value of each population using reproduction operation. During crossover operation, the randomly selected mating pairs exchange properties between the two parents to form two children solutions. Mutation operation is used to avoid the local minima problem, which brings a local change to the solution. The sequence of these steps will repeat until the termination criteria met [13]. In the present attempt of maximization of benzyl acetate molar conversion (\%), utilizing the duality concept the maximization problem was converted to minimization problem. An overall 40 bits (10-bits of each variable) GA-string was utilized in the search pattern.

\section{Results and Discussion}

In lipase-catalyzed transesterification reactions, different transesterification variables, namely, molarity of alcohol, reaction time, temperature, and enzyme amount play an important role on the final molar conversion [4]. In this study, central composite design of RSM including five factors with three levels was used to obtain a proper model for the immobilized lipase-mediated synthesis of flavour esters.

3.1. Model Development, Statistical Analysis, and Validation. Modeling task of immobilized lipase-mediated solvent-free synthesis of benzyl acetate has been carried out through running of suggested set of experimental runs of central composite design. The complete set of 27 experimental design matrix and its responses based on experimental runs and its predicted values of molar conversion proposed by CCD are given in Table 2, which facilitates in evaluating relationship between controllable experimental factors and observed results [23]. Based on these results, an empirical relationship between the responses and independent variables of immobilized lipase-catalyzed biosynthesis of benzyl acetate has been expressed using the following second-order polynomial equation in coded form:

$$
\begin{aligned}
\operatorname{MC}(\%)= & 94.9260-0.4533 X_{1}+3.3772 X_{2} \\
& -0.3994 X_{3}+5.4272 X_{4}-0.2244 X_{1} X_{2} \\
& +0.5719 X_{1} X_{3}-0.4806 X_{1} X_{4}-0.5044 X_{2} X_{3} \\
& -2.5869 X_{2} X_{4}-0.3331 X_{3} X_{4}-2.1741 X_{1}^{2} \\
& -4.8891 X_{2}^{2}-1.8891 X_{3}^{2}-6.8891 X_{4}^{2},
\end{aligned}
$$

where $X_{1}, X_{2}, X_{3}$ and $X_{4}$ represent the input process namely molarity of alcohol $(\mathrm{M})$, reaction time $(\mathrm{h})$, temperature $\left({ }^{\circ} \mathrm{C}\right)$, and immobilized enzyme amount (U), respectively.

The $P$ values of significance test (Table 3 ) for $X_{2}, X_{4}$, $X_{1}^{2}, X_{2}^{2}, X_{4}^{2}$ and $X_{2} X_{4}$, are found to be less than 0.05 and are considered to have significant impact on the molar conversion (\%) by considering $95 \%(a=0.05)$ as a level of confidence. The $P$ value of the factors $X_{1}, X_{3}, X_{3}^{2}, X_{1} X_{2}$, $X_{1} X_{3}, X_{1} X_{4}, X_{2} X_{3}$ and $X_{3} X_{4}$ is found to be more than the confidence level (0.05), but their square terms $P$ value is found to be less than the confidence level which indicates its nonlinear relationship with the response. The significant contribution of the linear, square, and interaction terms towards the response has been also depicted through the ANOVA results (Table 4 ), where the $P$ values were seen to be less than the significance value level $(\alpha 0.05)$ for all terms. Moreover, the good prediction accuracy and generalization ability of the predicted model has been visualized through close agreement of predicted and experimental values of the RSM design. The coefficient of multiple regression $R^{2}$ values and the adjusted $R^{2}$ values was found to be $98.6 \%$, and $97.1 \%$ respectively, which indicate the fitness and adequacy of the model [24]. As there is no dramatic difference between $R^{2}$ and adj $R^{2}$, it can be assumed that the nonsignificant terms have not been included in the model. Furthermore, the analysis of deleted residuals showed that the deleted residuals were well within the acceptable limits of +3 and -3 with only one observation beyond the acceptable limits which can be considered as a case of outlier (Table 2).

The response surface plots (Figure 1) were analyzed to understand the interaction and influence of different transesterification variables on the molar conversion (\%) of flavour ester. The response surface plots in Figures 1(a), 1(c), and 1(d) were both part of a parabolic cylinder, exhibiting a minimum and maximum ridge, respectively, in the investigated domain which indicates the nonlinear relationship of interaction variables on molar conversion. In these plots, the optimum values of both variable factors, such as the reaction time $\&$ alcohol molarity (Figure 1(a)), enzyme activity \& alcohol molarity (Figure $1(\mathrm{c})$ ), and enzyme activity \& temperature (Figure 1(f)) could be analyzed by the saddle point or by determining the maxima formed by the $x$ - and $y$-coordinates. Interaction effects of temperature with alcohol molarity \& reaction time and enzyme activity \& reaction time on molar 
TABLE 2: Central composite design with the experimental, predicted responses and its $R$-studentized residuals.

\begin{tabular}{|c|c|c|c|c|c|c|c|}
\hline \multirow{2}{*}{ Run order } & \multicolumn{4}{|c|}{ Input variables } & \multicolumn{3}{|c|}{ Response (MC(\%)) } \\
\hline & MA & RT & Temp & EA & Exp. & Predicted & $R$-studentized residual \\
\hline 1 & 0.3 & 12 & 40 & 12 & 90.98 & 93.21 & -2.49 \\
\hline 2 & 0.7 & 8 & 30 & 8 & 64.96 & 66.94 & -2.77 \\
\hline 3 & 0.3 & 16 & 30 & 8 & 80.67 & 80.96 & -0.32 \\
\hline 4 & 0.5 & 12 & 40 & 12 & 96.00 & 94.93 & 0.74 \\
\hline 5 & 0.7 & 16 & 50 & 8 & 78.00 & 79.43 & -1.73 \\
\hline 6 & 0.3 & 16 & 50 & 8 & 79.56 & 78.68 & 0.99 \\
\hline 7 & 0.5 & 12 & 40 & 8 & 82.00 & 82.61 & -0.55 \\
\hline 8 & 0.7 & 8 & 50 & 8 & 70.24 & 68.96 & 1.52 \\
\hline 9 & 0.3 & 8 & 50 & 16 & 83.52 & 83.63 & -0.12 \\
\hline 10 & 0.5 & 12 & 50 & 12 & 92.00 & 92.64 & -0.57 \\
\hline 11 & 0.3 & 8 & 30 & 16 & 85.46 & 85.23 & 0.24 \\
\hline 12 & 0.3 & 16 & 30 & 16 & 88.45 & 88.27 & 0.19 \\
\hline 13 & 0.5 & 12 & 40 & 12 & 96.00 & 94.93 & 0.74 \\
\hline 14 & 0.7 & 16 & 50 & 16 & 84.00 & 83.48 & 0.57 \\
\hline 15 & 0.7 & 8 & 50 & 16 & 82.45 & 83.36 & -1.02 \\
\hline 16 & 0.5 & 12 & 40 & 12 & 96.00 & 94.93 & 0.74 \\
\hline 17 & 0.5 & 16 & 40 & 12 & 92.00 & 93.41 & -1.37 \\
\hline 18 & 0.5 & 12 & 40 & 16 & 93.00 & 93.47 & -0.42 \\
\hline 19 & 0.5 & 8 & 40 & 12 & 87.00 & 86.66 & 0.30 \\
\hline 20 & 0.7 & 16 & 30 & 8 & 81.00 & 79.42 & 1.97 \\
\hline 21 & 0.3 & 8 & 30 & 8 & 68.52 & 67.58 & 1.07 \\
\hline 22 & 0.5 & 12 & 30 & 12 & 93.00 & 93.44 & -0.39 \\
\hline 23 & 0.3 & 16 & 50 & 16 & 85.43 & 84.65 & 0.86 \\
\hline 24 & 0.7 & 8 & 30 & 16 & 83.25 & 82.67 & 0.63 \\
\hline 25 & 0.3 & 8 & 50 & 8 & 66.92 & 67.31 & -0.42 \\
\hline 26 & 0.7 & 16 & 30 & 16 & 84.00 & 84.81 & -0.90 \\
\hline 27 & 0.7 & 12 & 40 & 12 & 93.45 & 92.29 & 1.08 \\
\hline
\end{tabular}

TABLE 3: Results of significance test on the nonlinear model coefficients, standard errors, $T$ statistics, and $P$ values for the lipase activity (coded form).

\begin{tabular}{|c|c|c|c|c|c|}
\hline SI. no & Terms & Standard coefficient & Error coefficient & $T$ & $P$ \\
\hline 1 & Constant & 94.9260 & 0.5609 & 169.225 & 0.000 \\
\hline 2 & $X_{1}$ & -0.4533 & 0.3588 & -1.264 & 0.230 \\
\hline 3 & $X_{2}$ & 3.3772 & 0.3588 & 9.413 & 0.000 \\
\hline 4 & $X_{3}$ & -0.3994 & 0.3588 & -1.113 & 0.287 \\
\hline 5 & $X_{4}$ & 5.4272 & 0.3588 & 15.127 & 0.000 \\
\hline 6 & $X_{1}^{2}$ & -2.1741 & 0.9492 & -2.290 & 0.041 \\
\hline 7 & $X_{2}^{2}$ & -4.8891 & 0.9492 & -5.150 & 0.000 \\
\hline 8 & $X_{3}^{2}$ & -1.8891 & 0.9492 & -1.990 & 0.070 \\
\hline 9 & $X_{4}^{2}$ & -6.8891 & 0.9492 & -7.257 & 0.000 \\
\hline 10 & $X_{1} X_{2}$ & -0.2244 & 0.3805 & -0.590 & 0.566 \\
\hline 11 & $X_{1} X_{3}$ & 0.5719 & 0.3805 & 1.503 & 0.159 \\
\hline 12 & $X_{1} X_{4}$ & -0.4806 & 0.3805 & -1.263 & 0.231 \\
\hline 13 & $X_{2} X_{3}$ & -0.5044 & 0.3805 & -1.325 & 0.210 \\
\hline 14 & $X_{2} X_{4}$ & -2.5869 & 0.3805 & -6.798 & 0.000 \\
\hline 15 & $X_{3} X_{4}$ & -0.3331 & 0.3805 & -0.875 & 0.399 \\
\hline
\end{tabular}

$\mathrm{SS}=1.522, R-\mathrm{Sq}=98.6 \%, R-\mathrm{Sq}(\mathrm{adj})=97.1 \%$. 
TABLE 4: Results of ANNOVA-lipase activity.

\begin{tabular}{|c|c|c|c|c|c|c|}
\hline Source & DF & Sequential SS & Adjusted SS & Adjusted MS & $F$ & $P$ \\
\hline Regression & 14 & 2027.81 & 2027.81 & 144.844 & 62.51 & 0.000 \\
\hline Linear & 4 & 742.06 & 742.06 & 185.514 & 80.07 & 0.000 \\
\hline Square & 4 & 1163.10 & 1163.10 & 290.775 & 125.49 & 0.000 \\
\hline Interaction & 6 & 122.65 & 122.65 & 20.442 & 8.82 & 0.001 \\
\hline Residual error & 12 & 27.80 & 27.80 & 2.317 & & \\
\hline Lack of fit & 10 & 27.80 & 27.80 & 2.780 & & \\
\hline Pure error & 2 & 0.00 & 0.00 & 0.000 & & \\
\hline Total & 26 & 2055.62 & & & & \\
\hline
\end{tabular}

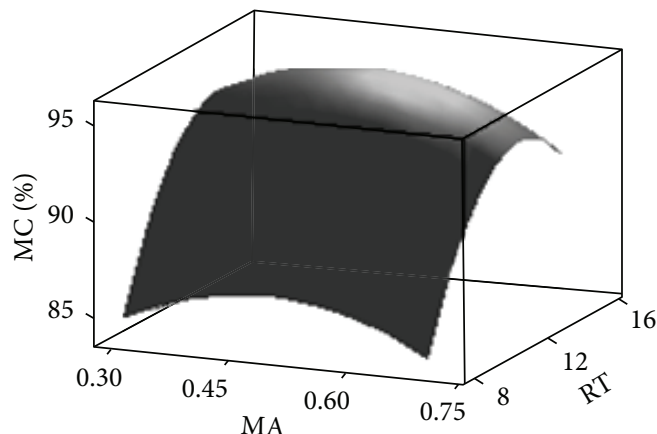

(a)

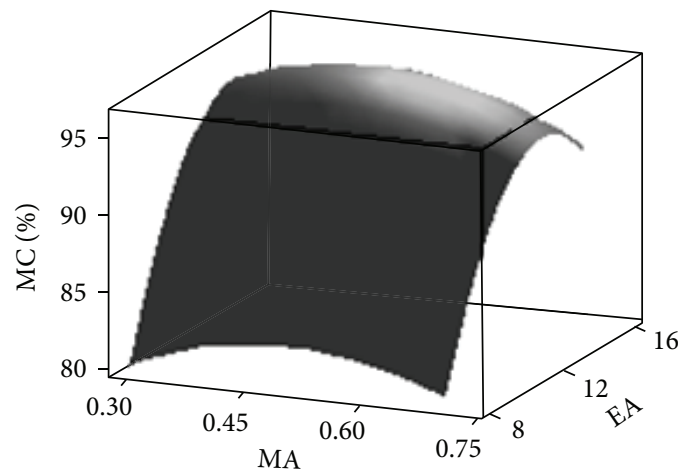

(c)

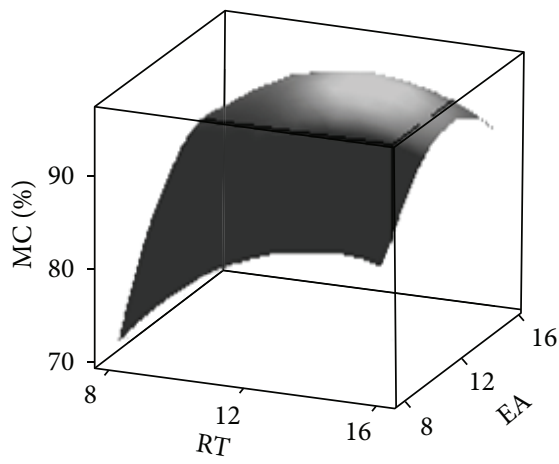

(e)

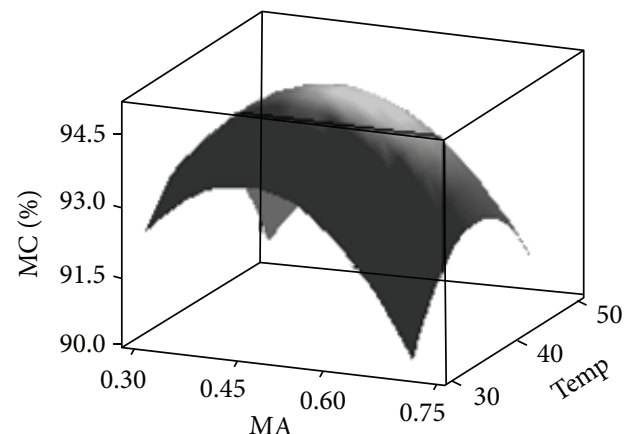

(b)

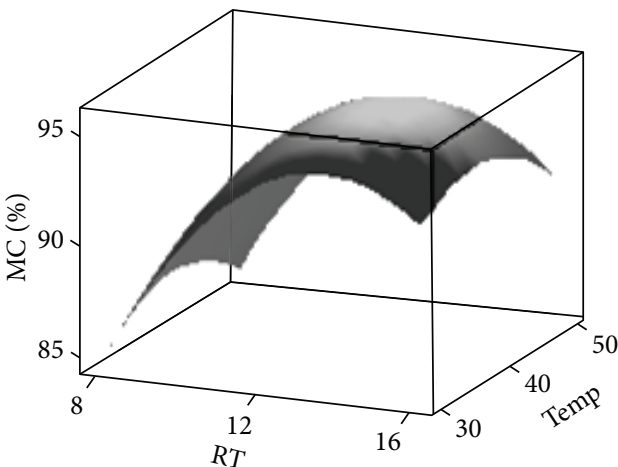

(d)

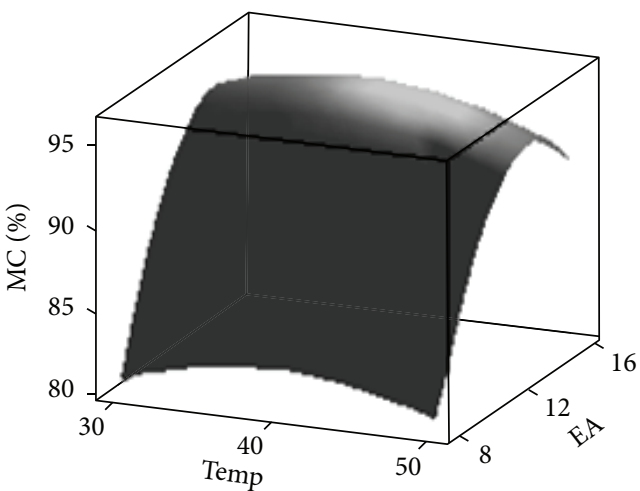

(f)

FIGURE 1: Surface plots of molar conversion (\%) with: (a) alcohol molarity and reaction time, (b) alcohol molarity and temperature, (c) alcohol molarity and enzyme activity, (d) reaction time and temperature, (e) reaction time and enzyme activity, (f) temperature and enzyme activity. 
conversion have been depicted through response surface plots of Figures 1(b), 1(d), and 1(e). The convex nature of these plots is clearly indicating the significance of variable interactions on the molar conversion. The response equation can be written in the uncoded form as follows:

$$
\begin{aligned}
\operatorname{MC} & (\%) \\
= & -111.473+51.2227 X_{1}+10.7627 X_{2} \\
& +1.57960 X_{3}+14.2641 X_{4}-0.280469 X_{1} X_{2} \\
& +0.285937 X_{1} X_{3}-0.600781 X_{1} X_{4}-0.0126094 X_{2} X_{3} \\
& -0.161680 X_{2} X_{4}-0.00832813 X_{3} X_{4}-54.3519 X_{1}^{2} \\
& -0.305567 X_{2}^{2}-0.0188907 X_{3}^{2}-0.430567 X_{4}^{2},
\end{aligned}
$$

where $X_{1}, X_{2}, X_{3}$, and $X_{4}$ represent the input process parameters of transesterification reaction.

The significant effect of temperature, reaction time, and enzyme amount on ester yield were also reported in case of lipase-mediated synthesis of flavour-active octanoic acid esters acetate [14]. Martins et al. 2011 [16] utilized central composite design of RSM for modeling the esterification reaction of butyl acetate synthesis catalyzed by Candida antarctica lipase B (Novozym 435) and reported the significance effects of transesterification variables such as temperature, alcohol molar ratio, and enzyme content on ester conversion yields. The obtained uncoded nonlinear response equation based on CCD has been taken as objective function for simulation studies of genetic algorithm for enhanced molar conversion (\%).

\subsection{Artificial Intelligence-Based Optimization Approach. The} present optimization approach of binary coded GA has been aimed to enhance the molar conversion of flavor ester synthesized through lipase-mediated transesterification reaction. Parameters such as population size and mutation constant take leading roles in search and selection aspects of the Darwanian evolution [25]. Applying GA in determining optimal molar conversion, tournament selection of size two, uniform crossover probability $(\mathrm{Pc}$ ) of 0.5 , bit-wise mutational probability (Pm) of 0.0001 , population size of 50, and maximum number of generations of 38 were employed. Executed parametric study in finding the optimal molar conversion of $96.41 \%$ and concerned results is depicted in Figure 2. The predicted transesterification variables were seen to be equal to $0.47 \mathrm{M}, 13.17 \mathrm{~h}, 37.58^{\circ} \mathrm{C}$, and $13.31 \mathrm{U}$ for molarity of alcohol, reaction time, temperature, and immobilized lipase amount, respectively. The optimized set of transesterification variables and the corresponding maximum molar conversion of flavor ester were tested in triplicate experimental runs using $0.47 \mathrm{M}$ molarity of alcohol and $13.31 \mathrm{U} 13.1 \mathrm{~h}$, at $37.5^{\circ} \mathrm{C}$ in $13.1 \mathrm{~h}$. Under these results, the molar conversion seen to be equal to $96.32 \%$ (10\% enhancement compared to one variable at a time selection approach), with the close agreement of GA-predicted value. Several researchers acknowledged the efficient searching abilities of artificial intelligence such as GA in different biochemical studies. Giordano et al. 2011 [26] reported the efficient problem solving approach of GA-coupled Plackett-Burman methodology in enzymatic hydrolysis of lignocellulosic residues. An optimized result has also been reported for the optimization of biocatalytic transglycosylation processes using GA approach [27]. Moreover the enhanced optimizing capability of genetic algorithm has been acknowledged in case of industrial enzymes production. Evolutionary optimization of extraction from fermented broth has been reported for fungal lipase production [28]. Similar enhanced results were reported in case of fermentation medium optimization for the production of glucansucrase [29] and halogenase enzymes [30].

3.3. Reusability of the Biocatalyst. At the end of each cycle, the immobilized lipases were removed from the reaction medium and washed with hexane to remove any substrate or product retained on the matrix. After drying at room temperature, the immobilized lipases were again introduced into fresh medium. The residual activity measured for the immobilized lipase after each cycle is shown in Figure 3. The remaining activity of immobilized lipase after 4 uses accounts for more than $98 \%$ of the initial activity, which reduces to $50 \%$ after 12 cycles of use. The development of an attractive biocatalyst requires high stability that allows repeated use of the catalytic material. The reduction in the conversion measured after the fifth use of immobilized lipase may be a consequence of a combined effect of deactivation or desorption of lipase and the loss of biocatalyst material due to high stirring speeds and repeated manipulation operations that is, filtration/drying/addition to a new substrate mixture [31]. KarraChâabouni et al. 2006 [32] reported that approximately 29\% of the initial activity was retained after 10 cycles of use for the hexyl acetate synthesis by immobilized Staphylococcus simulans lipase. The Rhizopus sp. lipase immobilized on celite retains its high activity only until the second cycle, while most of its activity was lost after four cycles [33].

3.4. Kinetics of Benzyl Acetate Synthesis. In the reaction, initially when the product concentrations are zero, the expression for initial reaction rate is

$$
v=\frac{V_{\max }}{1+\left(K m_{\mathrm{A}} /[\mathrm{A}]\right)+\left(K m_{\mathrm{C}} /[\mathrm{C}]\right)},
$$

where $v$ is the initial reaction rate, $V_{\max }$ is the maximum reaction rate, $[\mathrm{A}]$ and $[\mathrm{C}]$ are the respective concentrations of vinyl acetate and benzyl alcohol and $K m_{\mathrm{A}}$ and $K m_{\mathrm{C}}$ are the kinetic constants for the vinyl acetate and benzyl alcohol, respectively.

In this solvent-free reaction for benzyl acetate synthesis, the concentration of vinyl acetate can be regarded as constant. So the initial reaction rate equation can be expressed simply as

$$
v=\frac{V_{\max }[\mathrm{C}]}{[\mathrm{C}]+K m_{\mathrm{Capp}}},
$$

where $v$ is the initial reaction rate, $V_{\max }$ the maximum initial reaction rate and $K m_{\text {Capp }}$ the apparent Michaelis constant. 


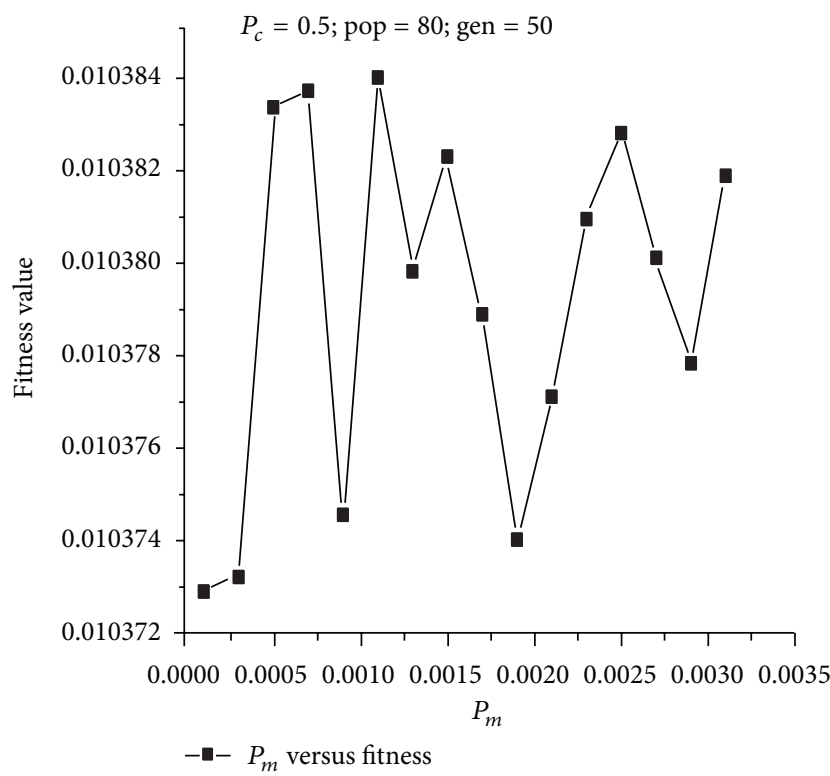

(a)

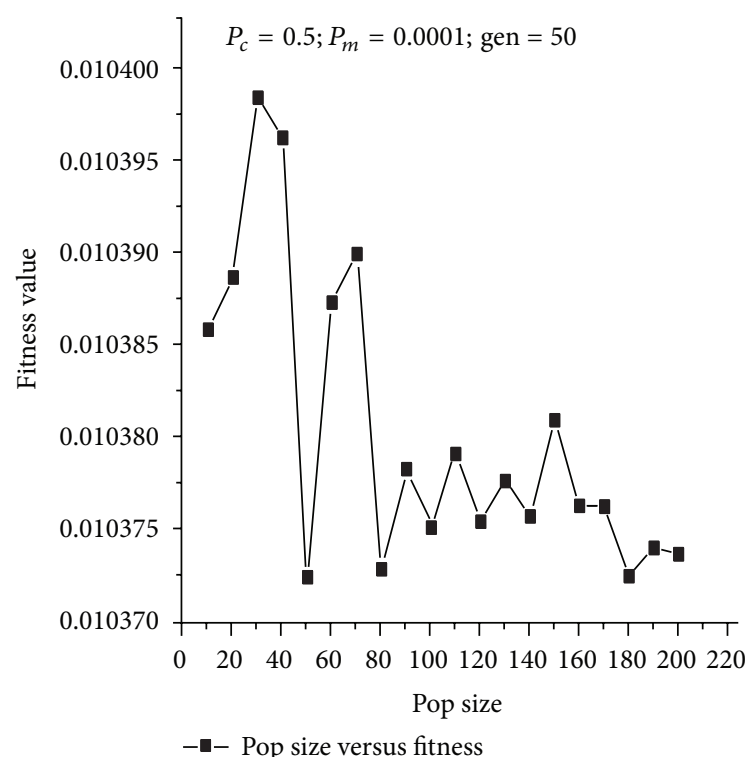

(b)

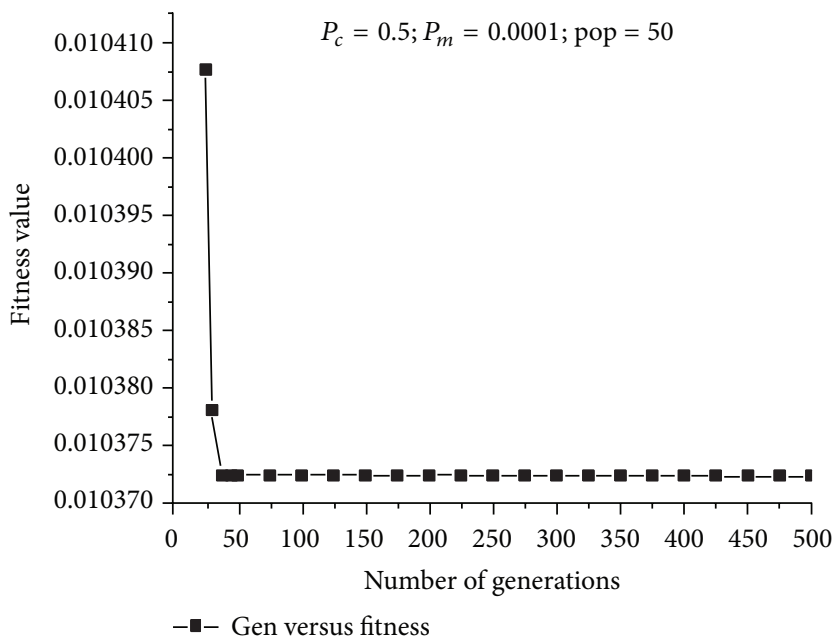

(c)

Figure 2: Results of parametric study. (a) Probability of mutation versus fitness, (b) population size versus fitness, (c) maximum number of generations versus fitness.

In the present investigation, kinetic parameters for benzyl acetate synthesis were calculated by varying the benzyl alcohol concentration (125-1000 mM). The kinetic constants $V_{\max }$ and $K m_{\text {Capp }}$ were calculated from the double reciprocal plot shown in Figure 4 . The $K_{m}$ and $V_{\max }$ value for benzyl acetate synthesis was $310 \mathrm{mM}$ and $0.10 \mathrm{mmol} \mathrm{h}^{-1} \mathrm{~g}^{-1}$, respectively. Previous studies have shown that the esterification and transesterification reactions using immobilized lipases could be described by the Ping-Pong kinetic models [34, 35]. As the synthesis reaction studied here was carried out at excess of vinyl acetate, so the concentration of vinyl acetate can be regarded as constant. Similar approaches have been applied previously for kinetic studies of lipase-mediated ester synthesis $[36,37]$.

\section{Conclusion}

In the present study, the individual and interaction effects of transesterification variables on molar conversion of benzyl acetate were studied and optimized through the statistical integrated artificial intelligence approach under solvent-free conditions catalyzed by immobilized lipase. The obtained results indicate that a maximal molar conversion of $96.32 \%$ was achieved in $13.17 \mathrm{~h}$ with the $0.47 \mathrm{M}$ benzyl alcohol in vinyl acetate and an immobilized enzyme amount of $13.31 \mathrm{U}$, at $37.58^{\circ} \mathrm{C}$ and $200 \mathrm{rpm}$. The immobilized lipase was reusable for four cycles with retaining the relative activity of more than $98 \%$. The kinetic parameters, $K_{m}$ and $V_{\max }$ values of the benzyl acetate synthesis were found to be $310 \mathrm{mM}$ 


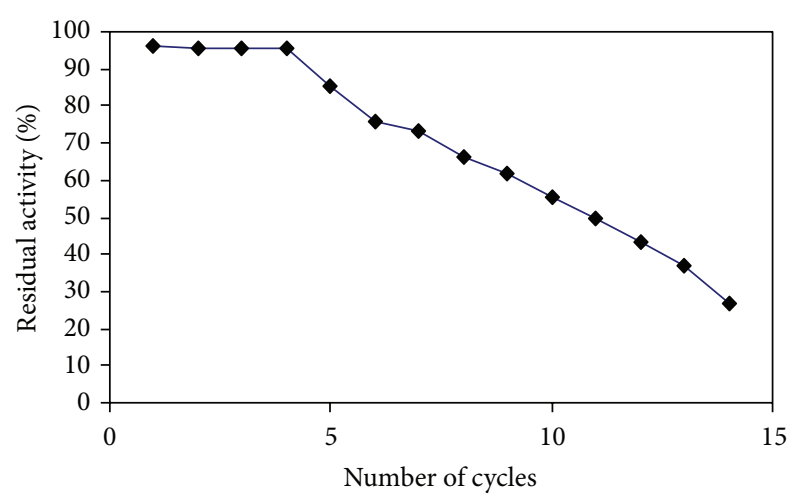

FIGURE 3: Operational stability of immobilized R. oryzae 3562 lipase for the synthesis of benzyl acetate.

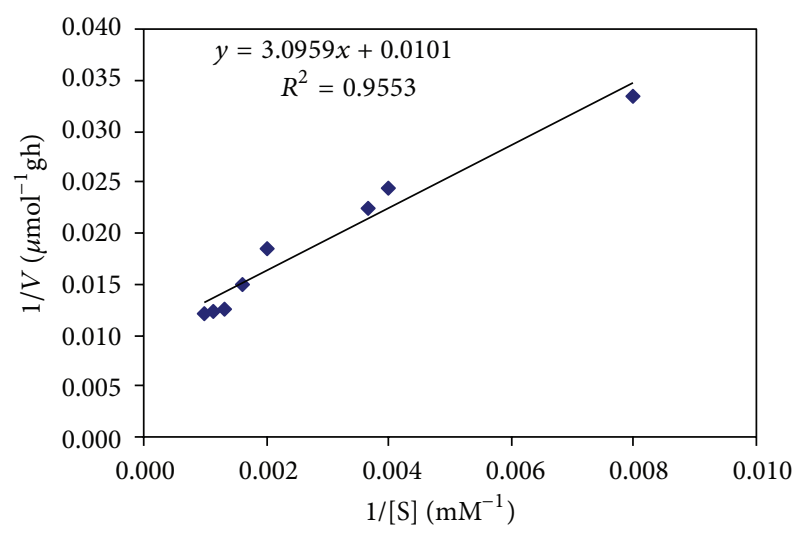

FIgURE 4: Lineweaver-Burk plots of immobilized R. oryzae 3562 lipase for benzyl acetate synthesis in solvent-free system.

and $0.10 \mathrm{mmol} \mathrm{h}^{-1} \mathrm{~g}^{-1}$, respectively. The results indicate that the statistical integrated artificial intelligence aspect was an efficient and systematic approach for optimization of immobilized lipase-catalyzed flavour ester synthesis.

\section{Authors' Contribution}

Vijay Kumar Garlapati and Annapurna Kumari equally contributed as a first author.

\section{Acknowledgments}

The authors gratefully acknowledge CSIR and MHRD, Govt of India and Department of Biotechnology, India for providing research fellowship to Annapurna Kumari and Vijay Kumar Garlapati, respectively.

\section{References}

[1] R. G. Berger, "Biotechnology of flavours-the next generation," Biotechnology Letters, vol. 31, no. 11, pp. 1651-1659, 2009.

[2] Y. C. Park, C. E. H. Shaffer, and G. N. Bennett, "Microbial formation of esters," Applied Microbiology and Biotechnology, vol. 85, no. 1, pp. 13-25, 2009.
[3] A. Wolfson, A. Atyya, C. Dlugy, and D. Tavor, "Glycerol triacetate as solvent and acyl donor in the production of isoamyl acetate with Candida antarctica lipase B," Bioprocess and Biosystems Engineering, vol. 33, no. 3, pp. 363-366, 2010.

[4] P. Mahapatra, A. Kumari, G. V. Kumar, R. Banerjee, and A. Nag, "Kinetics of solvent-free geranyl acetate synthesis by Rhizopus oligosporus NRRL 5905 lipase immobilized on to cross-linked silica," Biocatalysis and Biotransformation, vol. 27, no. 2, pp. 124130, 2009.

[5] A. Kumari, P. Mahapatra, V. K. Garlapati, R. Banerjee, and S. Dasgupta, "Lipase mediated isoamyl acetate synthesis in solvent-free system using vinyl acetate as acyl donor," Food Technology and Biotechnology, vol. 47, no. 1, pp. 13-18, 2009.

[6] X. Zhu, T. Zhou, X. Wu et al., "Covalent immobilization of enzymes within micro-aqueous organic media," Journal of Molecular Catalysis B, vol. 72, no. 3-4, pp. 145-149, 2011.

[7] C. A. Amarnath, S. Palaniappan, and C. Saravanan, "Preparation of benzyl acetate using polyaniline salts as catalysts-part II," Polymers for Advanced Technologies, vol. 15, no. 3, pp. 118121, 2004.

[8] A. B. Majumder, B. Singh, D. Dutta, S. Sadhukhan, and M. N. Gupta, "Lipase catalyzed synthesis of benzyl acetate in solventfree medium using vinyl acetate as acyl donor," Bioorganic and Medicinal Chemistry Letters, vol. 16, no. 15, pp. 4041-4044, 2006.

[9] J. E. S. Silva and P. C. Jesus, "Evaluation of the catalytic activity of lipases immobilized on chrysotile for esterification," Anais da Academia Brasileira de Ciencias, vol. 75, no. 2, pp. 157-162, 2003.

[10] Y. Yeşiloǧlu, "Utilization of bentonite as a support material for immobilization of Candida rugosa lipase," Process Biochemistry, vol. 40, no. 6, pp. 2155-2159, 2005.

[11] V. K. Garlapati, P. R. Vundavilli, and R. Banerjee, "Evaluation of lipase production by genetic algorithm and particle swarm optimization and their comparative study," Applied Biochemistry and Biotechnology, vol. 162, no. 5, pp. 1350-1361, 2010.

[12] R. H. Myers, D. C. Montgomery, and C. M. Anderson-Cook, Response Surface Methodology: Process and Product Optimization Using Designed Experiments, John Wiley \& Sons, Hoboken, NJ, USA, 2009.

[13] J. H. Holland, Adaptation in Natural and Artificial Systems, The MIT Press, Cambridge, Mass, USA, 1992.

[14] J. Sun, B. Yu, P. Curran, and S. Liu, "Optimisation of flavour ester biosynthesis in an aqueous system of coconut cream and fusel oil catalysed by lipase," Food Chemistry, vol. 35, no. 4, pp. 27142720, 2012.

[15] C. Grosso, S. Ferreira-Dias, and P. Pires-Cabral, "Modelling and optimization of ethyl butyrate production catalysed by Rhizopus oryzae lipase," Journal of Food Engineering, vol. 115, no. 4, pp. 475-480, 2012.

[16] A. B. Martins, N. G. Graebin, A. S. G. Lorenzoni, R. FernandezLafuente, M. A. Z. Ayub, and R. C. Rodrigues, "Rapid and high yields of synthesis of butyl acetate catalyzed by Novozym 435: reaction optimization by response surface methodology," Process Biochemistry, vol. 46, no. 12, pp. 2311-2316, 2011.

[17] P. Žnidaršič-Plazl and I. Plazl, "Modelling and experimental studies on lipase-catalyzed isoamyl acetate synthesis in a microreactor," Process Biochemistry, vol. 44, no. 10, pp. 1115-1121, 2009.

[18] V. K. Garlapati and R. Banerjee, "Optimization of lipase production using differential evolution," Biotechnology and Bioprocess Engineering, vol. 15, no. 2, pp. 254-260, 2010.

[19] A. Kumari, P. Mahapatra, G. V. Kumar, and R. Banerjee, "Comparative study of thermostabilty and ester synthesis ability of 
free and immobilized lipases on cross linked silica gel," Bioprocess and Biosystems Engineering, vol. 31, no. 4, pp. 291-298, 2008.

[20] P. Mahapatra, A. Kumari, V. K. Garlapati, R. Banerjee, and A. Nag, "Optimization of process variables for lipase biosynthesis from Rhizopus oligosporus NRRL 5905 Using evolutionary operation factorial design technique," Indian Journal of Microbiology, vol. 50, no. 4, pp. 396-403, 2010.

[21] O. H. Lowry, N. J. Rosebrough, A. L. Farr, and R. J. Randall, "Protein measurement with the Folin phenol reagent," The Journal of biological chemistry, vol. 193, no. 1, pp. 265-275, 1951.

[22] M. A. Bezerra, R. E. Santelli, E. P. Oliveira, L. S. Villar, and L. A. Escaleira, "Response surface methodology (RSM) as a tool for optimization in analytical chemistry," Talanta, vol. 76, no. 5, pp. 965-977, 2008.

[23] D. C. Montgomery and G. C. Runger, Applied Statistics and Probability For Engineers, John Wiley \& Sons, Singapore, 2002.

[24] S. S. Bhattacharya, V. K. Garlapati, and R. Banerjee, "Optimization of laccase production using response surface methodology coupled with differential evolution," New Biotechnology, vol. 28, no. 1, pp. 31-39, 2011.

[25] D. E. Goldberg, Genetic Algorithms in Search, Optimization and Machine Learning, Addison-Wesley, Reading, Mass, USA, 1989.

[26] P. C. Giordano, A. J. Beccaria, and H. C. Goicoechea, "Significant factors selection in the chemical and enzymatic hydrolysis of lignocellulosic residues by a genetic algorithm analysis and comparison with the standard Plackett-Burman methodology," Bioresource Technology, vol. 102, no. 22, pp. 10602-10610, 2011.

[27] R. Beine, A. R. Valente, R. Biedendieck, D. Jahn, and J. Seibel, "Directed optimization of biocatalytic transglycosylation processes by the integration of genetic algorithms and fermentative approaches into a kinetic model," Process Biochemistry, vol. 44, no. 10, pp. 1103-1114, 2009.

[28] V. K. Garlapati and R. Banerjee, "Evolutionary and swarm intelligence-based approaches for optimization of lipase extraction from fermented broth," Engineering in Life Sciences, vol. 10, no. 3, pp. 265-273, 2010.

[29] A. Singh, A. Majumder, and A. Goyal, "Artificial intelligence based optimization of exocellular glucansucrase production from Leuconostoc dextranicum NRRL B-1146," Bioresource Technology, vol. 99, no. 17, pp. 8201-8206, 2008.

[30] K. Muffler, M. Retzlaff, K.-H. van Pée, and R. Ulber, “Optimisation of halogenase enzyme activity by application of a genetic algorithm," Journal of Biotechnology, vol. 127, no. 3, pp. 425-433, 2007.

[31] M. L. Foresti and M. L. Ferreira, "Solvent-free ethyl oleate synthesis mediated by lipase from Candida antarctica B adsorbed on polypropylene powder," Catalysis Today, vol. 107-108, pp. 2330, 2005.

[32] M. Karra-Châabouni, H. Ghamgui, S. Bezzine, A. Rekik, and Y. Gargouri, "Production of flavour esters by immobilized Staphylococcus simulans lipase in a solvent-free system," Process Biochemistry, vol. 41, no. 7, pp. 1692-1698, 2006.

[33] L. L. M. M. Melo, G. M. Pastore, and G. A. Macedo, "Optimized synthesis of citronellyl flavour esters using free and immobilized lipase from Rhizopus sp," Process Biochemistry, vol. 40, no. 10, pp. 3181-3185, 2005.

[34] W. Chulalaksananukul, J. S. Condoret, P. Delorme, and R. M. Willemot, "Kinetic study of esterification by immobilized lipase in n-hexane," FEBS Letters, vol. 276, no. 1-2, pp. 181-184, 1990.
[35] A. Marty, W. Chulalaksananukul, R. M. Willemot, and J. S. Condoret, "Kinetics of lipase-catalyzed esterification in supercritical $\mathrm{CO}_{2}$," Biotechnology and Bioengineering, vol. 39, no. 3, pp. 273280, 1992.

[36] L. Tang, Z. Hao, M. M. Shehate, and Y. Sun, "A kinetic study of the synthesis of ascorbate fatty acid esters catalysed by immobilized lipase in organic media," Biotechnology and Applied Biochemistry, vol. 32, no. 1, pp. 35-39, 2000.

[37] Q.-X. Song and D.-Z. Wei, "Study of Vitamin C ester synthesis by immobilized lipase from Candida sp," Journal of Molecular Catalysis B, vol. 18, no. 4-6, pp. 261-266, 2002. 

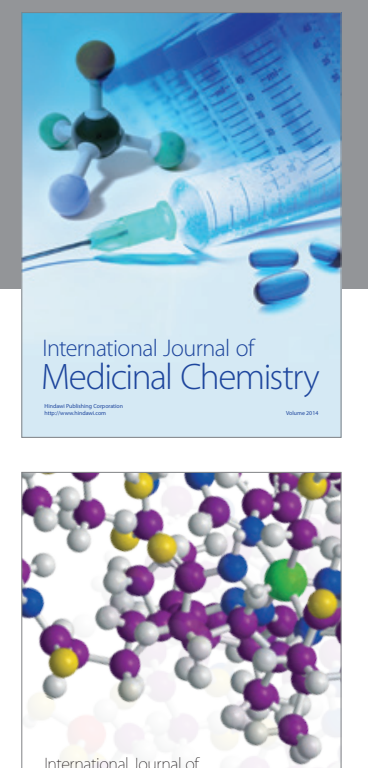

\section{Carbohydrate} Chemistry

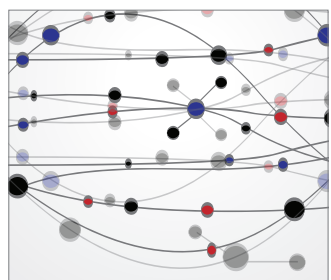

The Scientific World Journal
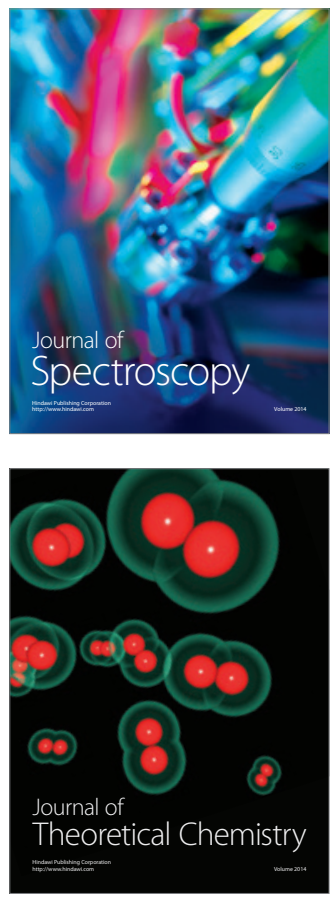
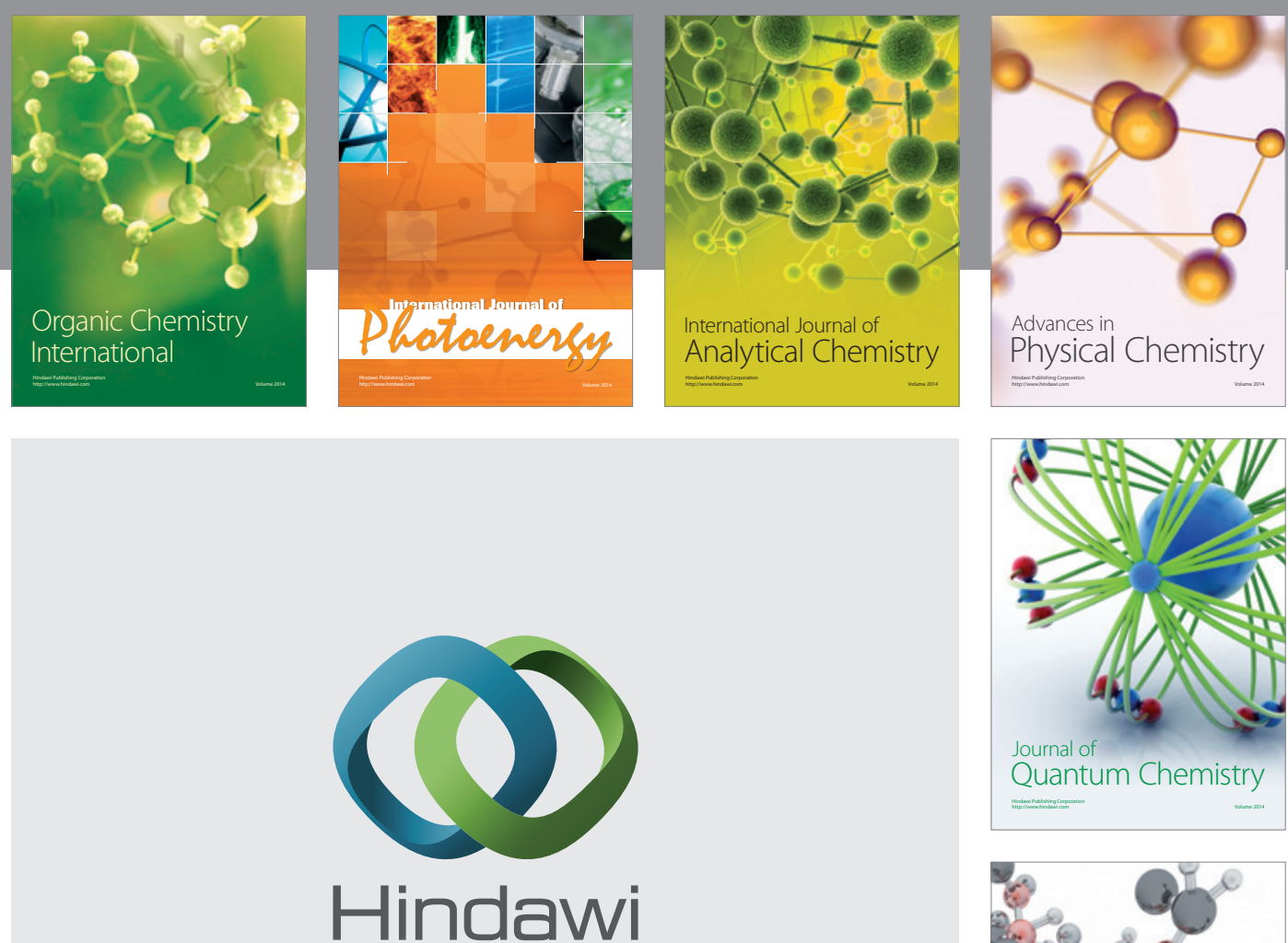

Submit your manuscripts at

http://www.hindawi.com

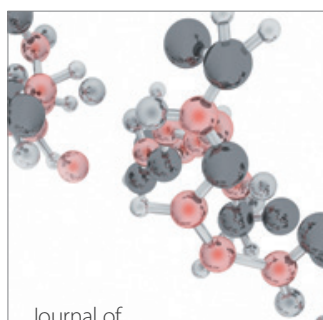

Analytical Methods

in Chemistry

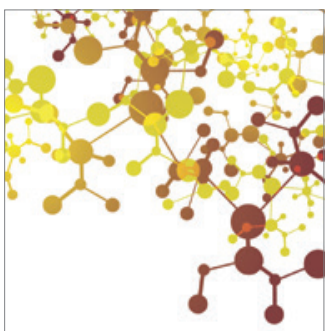

Journal of

Applied Chemistry

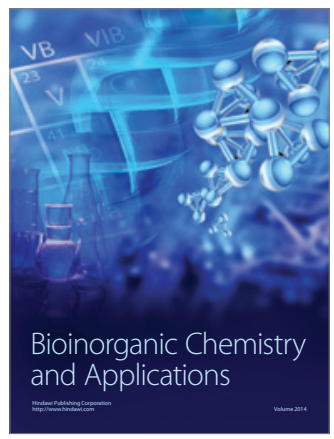

Inorganic Chemistry
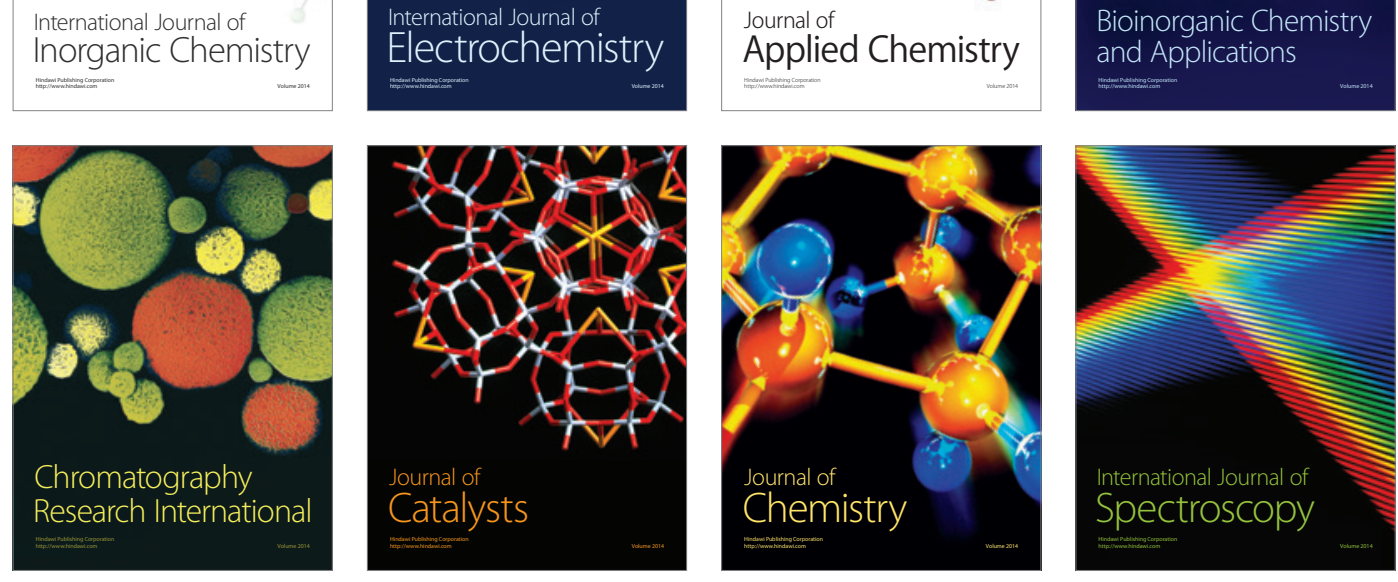